Kragujevac Journal of Mathematics

Volume 42(2) (2018), Pages 275-285.

\title{
CONCIRCULAR CURVATURE TENSOR ON A P-SASAKIAN MANIFOLD ADMITTING A QUARTER-SYMMETRIC METRIC CONNECTION
}

\author{
A. BARMAN
}

\begin{abstract}
The object of the present paper is to study a Para-Sasakian manifold admitting a type of quarter-symmetric metric connection whose concircular curvature tensor satisfies certain curvature conditions.
\end{abstract}

\section{INTRODUCTION}

In 1977, Adati and Matsumoto [1] defined Para-Sasakian and Special Para-Sasakian manifolds which are special classes of an almost paracontact manifold introduced by Sato [14]. Para-Sasakian manifolds have been studied by De and Pathak [6], Matsumoto, Ianus and Mihai [13], De, Özgür, Arslan, Murathan and Yildiz [7], Desmukh and Ahmed [8], Yildiz, Turan and Acet [17], Barman ([2], [3]) and many others.

In 1924, Friedmann and Schouten [9] introduced the idea of semi-symmetric connection on a differentiable manifold. A linear connection $\tilde{\nabla}$ on a differentiable manifold $M$ is said to be a semi-symmetric connection if the torsion tensor $T$ of the connection $\tilde{\nabla}$ satisfies $T(X, Y)=u(Y) X-u(X) Y$, where $u$ is a 1 -form and $\rho$ is a vector field defined by $u(X)=g(X, \rho)$, for all vector fields $X$ on $\chi(M), \chi(M)$ is the set of all differentiable vector fields on $M$.

In 1975, Golab [10] defined and studied quarter-symmetric connection in differentiable manifolds with affine connections. A liner connection $\bar{\nabla}$ on an $n$-dimensional Riemannian manifold $(M, g)$ is called a quarter-symmetric connection [10] if its torsion tensor $T$ satisfies $T(X, Y)=u(Y) \phi X-u(X) \phi Y$, where $\phi$ is a $(1,1)$ tensor field.

\footnotetext{
Key words and phrases. Para-Sasakian manifold, quarter-symmetric metric connection, concircular curvature tensor, $\xi$-concircularly flat, $\phi$-concircularly flat, $\eta$-Einstein manifold.

2010 Mathematics Subject Classification. Primary: 53C15. Secondary: 53C25.

Received: January 25, 2017.

Accepted: March 06, 2017.
} 
In particular, if $\phi X=X$, then the quarter-symmetric connection reduces to the semi-symmetric connection [9]. Thus the notion of the quarter-symmetric connection generalizes the notion of the semi-symmetric connection.

If moreover, a quarter-symmetric connection $\bar{\nabla}$ satisfies the condition

$$
\left(\bar{\nabla}_{X} g\right)(Y, Z)=0
$$

for all $X, Y, Z$ on $\chi(M)$, then $\bar{\nabla}$ is said to be a quarter-symmetric metric connection .

In a recent paper Mandal and De [12] studied a type of quarter-symmetric metric connection $\bar{\nabla}$ and it is given by

$$
\bar{\nabla}_{X} Y=\nabla_{X} Y+\eta(Y) \phi X-g(\phi X, Y) \xi
$$

where $\nabla$ is the Levi-Civita connection and $\xi$ is the corresponding vector field metrically equivalent to the $1-$ form $\eta$ defined by $\eta(X)=g(X, \xi)$.

Let $\bar{R}$ and $R$ be the curvature tensors with respect to the quarter-symmetric metric connection $\bar{\nabla}$ and the Levi-Civita connection $\nabla$ respectively. Then we have from [12],

$$
\begin{aligned}
\bar{R}(X, Y) Z= & R(X, Y) Z+3 g(\phi X, Z) \phi Y-3 g(\phi Y, Z) \phi X \\
& +\eta(Z)[\eta(X) Y-\eta(Y) X] \\
& -[\eta(X) g(Y, Z)-\eta(Y) g(X, Z)] \xi, \\
\bar{S}(Y, U)= & S(Y, U)+2 g(Y, U)-(n+1) \eta(Y) \eta(U) \\
& -3 \operatorname{trace} \phi \quad g(\phi Y, U), \\
\bar{S}(Y, \xi)= & -2(n-1) \eta(Y)
\end{aligned}
$$

and

$$
\bar{R}(\xi, Y) U=2[\eta(U) Y-g(U, Y) \xi],
$$

where $\bar{S}$ and $S$ be the Ricci tensors with respect to the quarter-symmetric metric connection $\bar{\nabla}$ and the Levi-Civita connection $\nabla$ respectively.

A transformation of an n-dimensional Riemannian manifold $M$, which transforms every geodesic circle of $M$ into a geodesic circle, is called a concircular transformation ([11], [15] ). A concircular transformation is always a conformal transformation [11]. Here geodesic circle means a curve in $M$ whose first curvature is constant and whose second curvature is identically zero. Thus the geometry of concircular transformations, i.e., the concircular geometry, is a generalization of inversive geometry in the sense that the change of metric is more general than that induced by a circle preserving diffeomorphism (see also [5]). An interesing invariant of a concircular transformation is the concircular curvature tensor $\overline{\mathbb{W}}$. It is defined by $([15],[16])$

$$
\overline{\mathbb{W}}(X, Y) Z=\bar{R}(X, Y) Z-\frac{\bar{r}}{n(n-1)}[g(Y, Z) X-g(X, Z) Y] .
$$


From (1.6), it follows that

$$
\begin{aligned}
\widetilde{\mathbb{W}}(X, Y, Z, U)= & \widetilde{\bar{R}}(X, Y, Z, U)-\frac{\bar{r}}{n(n-1)}[g(Y, Z) g(X, U) \\
& -g(X, Z) g(Y, U)]
\end{aligned}
$$

and $\widetilde{\mathbb{W}}(X, Y, Z, U)=g(\overline{\mathbb{W}}(X, Y) Z, U) \widetilde{\bar{R}}(X, Y, Z, U)=g(\bar{R}(X, Y) Z, U)$, where $X, Y$, $Z, U$ on $M$ and $\overline{\mathbb{W}}$ is the concircular curvature tensor and $\bar{r}$ is the scalar curvature with respect to the quarter-symmetric metric connection respectively. Riemannian manifolds with vanishing concircular curvature tensor are of constant curvature. Thus the concircular curvature tensor is a measure of the failure of a Riemannian manifold to be of constant curvature.

Putting $X=\xi$ in (1.6) and using (1.5), we obtain

$$
\overline{\mathbb{W}}(\xi, Y) Z=\left[2+\frac{\bar{r}}{n(n-1)}\right][\eta(Z) Y-g(Y, Z) \xi] .
$$

A Riemannian manifold $M$ is locally symmetric if its curvature tensor $R$ satisfies $\nabla R=0$. As a generalization of locally symmetric spaces, many geometers have considered semi-symmetric spaces and in turn their generalizations. A Riemannian manifold $M$ is said to be semi-symmetric if its curvature tensor $R$ satisfies $R(X, Y) \cdot R=0$, where $R(X, Y)$ acts on $R$ as a derivation. A Riemannian manifold $M$ is said to be Ricci-semisymmetric manifold if the relation $\bar{R}(X, Y) \cdot \bar{S}=0$ holds, where $\bar{R}(X, Y)$ is the curvature operator.

In this paper we study a type of quarter-symmetric metric connection due to Mandal and De [12] on P-Sasakian manifolds. The paper is organized as follows: After introduction Section 2 is equipped with some prerequisites of a P-Sasakian manifold. Section 3 is devoted to study $\xi$-concircularly flat in a P-Sasakian manifold with respect to the quarter-symmetric metric connection. $\phi$-concircularly flat PSasakian manifolds with respect to the quarter-symmetric metric connection have been studied in Section 4. Next section we investigate Ricci-semisymmetric manifolds with respect to the quarter-symmetric metric connection of a P-Sasakian manifold. Finally, we construct an example of a 5-dimensional P-Sasakian manifolds admitting the quarter-symmetric metric connections which verify the results of Section 3 and Section 4.

\section{P-SASAKIAN MANIFOLDS}

An $n$-dimensional differentiable manifold $M$ is said to be an almost para-contact structure $(\phi, \xi, \eta, g)$,if there exists $\phi$ is a $(1,1)$ tensor field, $\xi$ is a vector field, $\eta$ is a 1 -form and $g$ is the Riemannian metric on $M$ which satisfy the conditions

$$
\begin{gathered}
\phi \xi=0, \quad \eta(\phi X)=0, \quad \eta(\xi)=1, \quad g(X, \xi)=\eta(X), \\
\phi^{2}(X)=X-\eta(X) \xi,
\end{gathered}
$$




$$
\begin{gathered}
g(\phi X, \phi Y)=g(X, Y)-\eta(X) \eta(Y), \\
\left(\nabla_{X} \eta\right) Y=g(X, \phi Y)=\left(\nabla_{Y} \eta\right) X
\end{gathered}
$$

for any vector fields $X, Y$ on $M$.

If moreover, $(\phi, \xi, \eta, g)$ satisfy the conditions

$$
\begin{gathered}
d \eta=0, \quad \nabla_{X} \xi=\phi X \\
\left(\nabla_{X} \phi\right) Y=-g(X, Y) \xi-\eta(Y) X+2 \eta(X) \eta(Y) \xi
\end{gathered}
$$

then $M$ is called a para-Sasakian manifold or briefly a P-Sasakian manifold.

In a P-Sasakian manifold the following relations hold ([1], [14]):

$$
\begin{aligned}
\eta(R(X, Y) Z) & =g(X, Z) \eta(Y)-g(Y, Z) \eta(X), \\
R(\xi, X) Y & =\eta(Y) X-g(X, Y) \xi, \\
R(\xi, X) \xi & =X-\eta(X) \xi, \\
R(X, Y) \xi & =\eta(X) Y-\eta(Y) X, \\
S(X, \xi) & =-(n-1) \eta(X), \\
S(\phi X, \phi Y) & =S(X, Y)+(n-1) \eta(X) \eta(Y),
\end{aligned}
$$

where $R$ and $S$ are the curvature tensor and the Ricci tensor of the Levi-Civita connection respectively.

\section{3. $\xi$-Concircularly Flat P-Sasakian Manifolds with Respect to the QuARTER-SYMmetric Metric Connection}

Definition 3.1. A P-Sasakian manifold is said to be $\xi$-concircularly flat [4] with respect to the quarter-symmetric metric connection if $\overline{\mathbb{W}}(X, Y) \xi=0$, where $X, Y \in$ $\chi(M)$.

Theorem 3.1. A P-Sasakian manifold admitting a quarter-symmetric metric connection is $\xi$-concircularly flat if and only if the scalar curvature $\bar{r}$ with respect to the quarter-symmetric metric connection is equal to $-2 n(n-1)$.

Proof. Combining (1.2) and (1.6), it follows that

$$
\begin{aligned}
\overline{\mathbb{W}}(X, Y,) Z= & R(X, Y) Z+3 g(\phi X, Z) \phi Y-3 g(\phi Y, Z) \phi X \\
& +\eta(Z)[\eta(X) Y-\eta(Y) X]-[\eta(X) g(Y, Z)-\eta(Y) g(X, Z)] \xi \\
& -\frac{\bar{r}}{n(n-1)}[g(Y, Z) X-g(X, Z) Y] .
\end{aligned}
$$

Putting $Z=\xi$ in (3.1) and using (2.1), we have

$$
\begin{aligned}
\overline{\mathbb{W}}(X, Y,) \xi= & R(X, Y) \xi+[\eta(X) Y-\eta(Y) X] \\
& +\frac{\bar{r}}{n(n-1)}[\eta(X) Y-\eta(Y) X] .
\end{aligned}
$$


By making use of (2.10) and (3.2), we get

$$
\overline{\mathbb{W}}(X, Y,) \xi=\left[2+\frac{\bar{r}}{n(n-1)}\right] R(X, Y) \xi .
$$

If $\overline{\mathbb{W}}(X, Y,) \xi=0$, then $\bar{r}=-2 n(n-1)$ or $R(X, Y) \xi=\eta(Y) X-\eta(X) Y=0$, implies that $\eta(X)=0$ which is not admissible.

Conversly, if $\bar{r}=-2 n(n-1)$, then from (3.3), it follows that $\overline{\mathbb{W}}(X, Y,) \xi=0$. This completes the proof.

Theorem 3.2. If P-Sasakian manifolds satisfying $\bar{R}(\xi, Y) \cdot \overline{\mathbb{W}}=0$ with respect to a quarter-symmetric metric connection, then the manifold is an Einstein manifold with respect to the quarter-symmetric metric connection and the scalar curvature $\bar{r}$ with respect to the quarter-symmetric metric connection is a negative constant.

Proof. Now we can state the following lemma.

Lemma 3.1. [12] If a P-Sasakian manifold is semisymmetric with respect to the quarter-symmetric metric connection, then the manifold is an Einstein manifold with respect to the quarter-symmetric metric connection and the scalar curvature $\bar{r}$ with respect to the quarter-symmetric metric connection is a negative constant.

From the definition of concircular curvature tensor, it follows that

$$
\bar{R}(X, Y) \cdot \overline{\mathbb{W}}=\bar{R}(X, Y) \cdot \bar{R}
$$

Thus, using Lemma 3.1 we obtain Theorem 3.2.

4. $\phi$-Concircularly Flat in P-Sasakian Manifolds with Respect to the QuARTER-SYMMETRIC Metric CONNECTION

Theorem 4.1. If a P-Sasakian manifold admitting a quarter-symmetric metric connection is $\phi$-concircularly flat, then the manifold with respect to the quarter-symmetric metric connection is an $\eta$-Einstein manifold.

Definition 4.1. A P-Sasakian manifold is said to be $\phi$-concircularly flat [4] with respect to the quarter-symmetric metric connection if $\widetilde{\mathbb{W}}(\phi X, \phi Y, \phi Z, \phi U)=0$, where $X, Y, Z, U \in \chi(M)$.

Definition 4.2. A P-Sasakian manifold is said to be an $\eta$-Einstein manifold if its Ricci tensor $S$ of the Levi-Civita connection is of the form $S(X, Y)=a g(X, Y)+b \eta(X) \eta(Y)$, where $\mathrm{a}$ and $\mathrm{b}$ are smooth functions on the manifold. 
Proof. In view of (1.2) and (1.7) yields

$$
\begin{aligned}
\widetilde{\widetilde{W}}(X, Y, Z, U)= & \widetilde{R}(X, Y, Z, U)+3 g(\phi X, Z) g(\phi Y, U)-3 g(\phi Y, Z) g(\phi X, U) \\
& +\eta(Z)[\eta(X) g(Y, U)-\eta(Y) g(X, U)] \\
& -[\eta(X) g(Y, Z)-\eta(Y) g(X, Z)] \eta(U) \\
& -\frac{\bar{r}}{n(n-1)}[g(Y, Z) g(X, U)-g(X, Z) g(Y, U)]
\end{aligned}
$$

where $\widetilde{R}(X, Y, Z, U)=g(R(X, Y) Z, U)$.

Now putting $X=\phi X, Y=\phi Y, Z=\phi Z, U=\phi U$ in (4.1) and using (2.1) and (2.2), we derive that

$$
\begin{aligned}
\widetilde{\widetilde{W}}(\phi X, \phi Y, \phi Z, \phi U)= & \widetilde{R}(\phi X, \phi Y, \phi Z, \phi U)+3 g(X, \phi Z) g(Y, \phi U) \\
& -3 g(Y, \phi Z) g(\phi X, \phi U) \\
& -\frac{\bar{r}}{n(n-1)}[g(Y, Z) g(X, U)-g(X, Z) g(Y, U)] .
\end{aligned}
$$

Let $\left\{e_{1}, \ldots, e_{n-1}, \xi\right\}$ be a local orthonormal basis of vector fields in $M$, then $\left\{\phi e_{1}, \ldots, \phi e_{n-1}, \xi\right\}$ is also a local orthonormal basis. Putting $X=U=e_{i}$ in (4.2) and summing over $i=1$ to $n-1$, we obtain

$$
\begin{aligned}
\widetilde{\mathbb{W}}\left(\phi e_{i}, \phi Y, \phi Z, \phi e_{i}\right)= & S(\phi Y, \phi Z)+3 g(Y, Z)-3 \alpha g(Y, \phi Z) \\
& -\frac{(n-2) \bar{r}}{n(n-1)} g(\phi Y, \phi Z),
\end{aligned}
$$

where $\alpha=g\left(\phi e_{i}, e_{i}\right)$.

Using (2.3) and (2.12) in (4.3), it follows that

$$
\begin{aligned}
\widetilde{\mathbb{W}}\left(\phi e_{i}, \phi Y, \phi Z, \phi e_{i}\right)= & S(Y, Z)+(n-1) \eta(Y) \eta(Z)+3 g(Y, Z)-3 \alpha g(Y, \phi Z) \\
& -\frac{(n-2) \bar{r}}{n(n-1)}[g(Y, Z)-\eta(Y) \eta(Z)] .
\end{aligned}
$$

By virtue of (1.3) and (4.4) yields

$$
\begin{aligned}
\widetilde{\mathbb{W}}\left(\phi e_{i}, \phi Y, \phi Z, \phi e_{i}\right)= & \bar{S}(Y, Z)+\left[1-\frac{(n-2) \bar{r}}{n(n-1)}\right] g(Y, Z) \\
& +\left[2 n+\frac{(n-2) \bar{r}}{n(n-1)}\right] \eta(Y) \eta(Z)
\end{aligned}
$$

If $\widetilde{\mathbb{W}}\left(\phi e_{i}, \phi Y, \phi Z, \phi e_{i}\right)=0$, then

$$
\bar{S}(Y, Z)=-\left[1-\frac{(n-2) \bar{r}}{n(n-1)}\right] g(Y, Z)-\left[2 n+\frac{(n-2) \bar{r}}{n(n-1)}\right] \eta(Y) \eta(Z),
$$

where $a=-\left[1-\frac{(n-2) \bar{r}}{n(n-1)}\right]$ and $b=2 n+\frac{(n-2) \bar{r}}{n(n-1)}$. 
From which it follows that the manifold is an $\eta$-Einstein manifold with respect to the quarter-symmetric metric connection. Hence the proof of Theorem 4.1 is completed.

5. P-Sasakian Manifolds Satisfying $\overline{\mathbb{W}} \cdot \bar{S}=0$ with Respect to a QuARTER-Symmetric Metric CONneCtion

Theorem 5.1. If P-Sasakian manifolds satisfying $\overline{\mathbb{W}} \cdot \bar{S}=0$ with respect to a quartersymmetric metric connection, then the manifold is an Einstein manifold with respect to a quarter-symmetric metric connection.

Proof. We consider P-Sasakian manifolds with respect to a quarter-symmetric metric connection $\bar{\nabla}$ satisfying the curvature condition $\overline{\mathbb{W}} \cdot \bar{S}=0$. Then

$$
(\overline{\mathbb{W}}(X, Y) \cdot \bar{S})(U, V)=0 \text {. }
$$

So,

$$
\bar{S}(\overline{\mathbb{W}}(X, Y) U, V)+\bar{S}(U, \overline{\mathbb{W}}(X, Y) V)=0 .
$$

Definition 5.1. A P-Sasakian manifold $(n>2)$ is said to be an Einstein manifold if its Ricci tensor $S$ of the Levi-Civita connection is of the form

$$
S(X, Y)=\tau g(X, Y),
$$

where $\tau$ is a constant on the manifold.

Putting $X=\xi$ in (5.1) and using (1.8), we get

$$
\begin{aligned}
& {\left[2+\frac{\bar{r}}{n(n-1)}\right][\eta(U) \bar{S}(Y, V)+2(n-1) \eta(V) g(U, Y)+\eta(V) \bar{S}(Y, U)} \\
+ & 2(n-1) \eta(U) g(V, Y)]=0 .
\end{aligned}
$$

Again putting $U=\xi$ in (5.2), implies that

$$
\bar{S}(Y, V)=-2(n-1) g(Y, V) .
$$

Therefore, $S(Y, Z)=\tau g(Y, Z)$, where $\tau=-2(n-1)$.

This means that the manifold is an Einstein manifold with respect to the quartersymmetric metric connection. This completes the proof.

\section{EXAMPLE}

In this section we construct an example on P-Sasakian manifold with respect to the quarter-symmetric metric connections $\bar{\nabla}[12]$ which verify the results of Section 3 and Section 4.

We consider the 5-dimensional manifold $\left\{(x, y, z, u, v) \in R^{5}\right\}$, where $(x, y, z, u, v)$ are the standard coordinates in $R^{5}$. We choose the vector fields

$$
e_{1}=\frac{\partial}{\partial x}, \quad e_{2}=e^{-x} \frac{\partial}{\partial y}, \quad e_{3}=e^{-x} \frac{\partial}{\partial z}, \quad e_{4}=e^{-x} \frac{\partial}{\partial u}, \quad e_{5}=e^{-x} \frac{\partial}{\partial v},
$$


which are linearly independent at each point of $M$. Let $g$ be the Riemannian metric defined by

$$
g\left(e_{i}, e_{j}\right)= \begin{cases}1, & \text { if } i=j \\ 0, & \text { if } i \neq j\end{cases}
$$

where $i, j=1,2,3,4,5$.

Let $\eta$ be the 1 -form defined by

$$
\eta(Z)=g\left(Z, e_{1}\right)
$$

for any $Z \in \chi(M)$.

Let $\phi$ be the $(1,1)$-tensor field defined by

$$
\phi\left(e_{1}\right)=0, \quad \phi\left(e_{2}\right)=e_{2}, \quad \phi\left(e_{3}\right)=e_{3}, \quad \phi\left(e_{4}\right)=e_{4}, \quad \phi\left(e_{5}\right)=e_{5} .
$$

Using the linearity of $\phi$ and $g$, we have

$$
\eta\left(e_{1}\right)=1, \quad \phi^{2} Z=Z-\eta(Z) e_{1}
$$

and

$$
g(\phi Z, \phi U)=g(Z, U)-\eta(Z) \eta(U),
$$

for any vector fields $Z$ and $U$ on $M$. Thus for $e_{1}=\xi$, the structure $(\phi, \xi, \eta, g)$ defines an almost paracontact metric structure on $M$. Then we have

$$
\begin{aligned}
& {\left[e_{1}, e_{2}\right]=-e_{2},\left[e_{1}, e_{3}\right]=-e_{3},\left[e_{1}, e_{4}\right]=-e_{4},\left[e_{1}, e_{5}\right]=-e_{5},} \\
& {\left[e_{2}, e_{3}\right]=\left[e_{2}, e_{4}\right]=0,\left[e_{2}, e_{5}\right]=\left[e_{3}, e_{4}\right]=\left[e_{3}, e_{5}\right]=\left[e_{4}, e_{5}\right]=0}
\end{aligned}
$$

The Levi-Civita connection $\nabla$ of the metric tensor $g$ is given by Koszul's formula which is given by

$$
\begin{aligned}
2 g\left(\nabla_{X} Y, Z\right)= & X g(Y, Z)+Y g(Z, X)-Z g(X, Y) \\
& -g(X,[Y, Z])-g(Y,[X, Z])+g(Z,[X, Y]) .
\end{aligned}
$$

Using Koszul's formula, we get the following:

$$
\begin{aligned}
& \nabla_{e_{1}} e_{1}=0, \nabla_{e_{1}} e_{2}=0, \nabla_{e_{1}} e_{3}=0, \nabla_{e_{1}} e_{4}=0, \nabla_{e_{1}} e_{5}=0, \\
& \nabla_{e_{2}} e_{1}=e_{2}, \nabla_{e_{2}} e_{2}=-e_{1}, \nabla_{e_{2}} e_{3}=0, \nabla_{e_{2}} e_{4}=0, \nabla_{e_{2}} e_{5}=0, \\
& \nabla_{e_{3}} e_{1}=e_{3}, \nabla_{e_{3}} e_{2}=0, \nabla_{e_{3}} e_{3}=-e_{1}, \nabla_{e_{3}} e_{4}=0, \nabla_{e_{3}} e_{5}=0, \\
& \nabla_{e_{4}} e_{1}=e_{4}, \nabla_{e_{4}} e_{2}=0, \nabla_{e_{4}} e_{3}=0, \nabla_{e_{4}} e_{4}=-e_{1}, \nabla_{e_{4}} e_{5}=0, \\
& \nabla_{e_{5}} e_{1}=e_{5}, \nabla_{e_{5}} e_{2}=0, \nabla_{e_{5}} e_{3}=0, \nabla_{e_{5}} e_{4}=0, \nabla_{e_{5}} e_{5}=-e_{1} .
\end{aligned}
$$

In view of the above relations, we see that $\nabla_{X} \xi=\phi X,\left(\nabla_{X} \phi\right) Y=-g(X, Y) \xi-$ $\eta(Y) X+2 \eta(X) \eta(Y) \xi$, for all $e_{5}=\xi$. 
Therefore the manifold is a P-Sasakian manifold with the structure $(\phi, \xi, \eta, g)[12]$. Using (1.1) in above equations, we obtain

$$
\begin{aligned}
& \bar{\nabla}_{e_{1}} e_{1}=0, \bar{\nabla}_{e_{1}} e_{2}=0, \bar{\nabla}_{e_{1}} e_{3}=0, \bar{\nabla}_{e_{1}} e_{4}=0, \bar{\nabla}_{e_{1}} e_{5}=0, \\
& \bar{\nabla}_{e_{2}} e_{1}=2 e_{2}, \bar{\nabla}_{e_{2}} e_{2}=-2 e_{1}, \bar{\nabla}_{e_{2}} e_{3}=0, \bar{\nabla}_{e_{2}} e_{4}=0, \bar{\nabla}_{e_{2}} e_{5}=0, \\
& \bar{\nabla}_{e_{3}} e_{1}=2 e_{3}, \bar{\nabla}_{e_{3}} e_{2}=0, \bar{\nabla}_{e_{3}} e_{3}=-2 e_{1}, \bar{\nabla}_{e_{3}} e_{4}=0, \bar{\nabla}_{e_{3}} e_{5}=0, \\
& \bar{\nabla}_{e_{4}} e_{1}=2 e_{4}, \bar{\nabla}_{e_{4}} e_{2}=0, \bar{\nabla}_{e_{4}} e_{3}=0, \bar{\nabla}_{e_{4}} e_{4}=-2 e_{1}, \bar{\nabla}_{e_{4}} e_{5}=0, \\
& \bar{\nabla}_{e_{5}} e_{1}=2 e_{5}, \bar{\nabla}_{e_{5}} e_{2}=0, \bar{\nabla}_{e_{5}} e_{3}=0, \bar{\nabla}_{e_{5}} e_{4}=0, \bar{\nabla}_{e_{5}} e_{5}=-2 e_{1} .
\end{aligned}
$$

Now, we can easily obtain the non-zero components of the curvature tensors [12] as follows:

$$
\begin{aligned}
& R\left(e_{1}, e_{2}\right) e_{1}=e_{2}, R\left(e_{1}, e_{2}\right) e_{2}=-e_{1}, R\left(e_{1}, e_{3}\right) e_{1}=e_{3}, R\left(e_{1}, e_{3}\right) e_{3}=-e_{1}, \\
& R\left(e_{1}, e_{4}\right) e_{1}=e_{4}, R\left(e_{1}, e_{4}\right) e_{4}=-e_{1}, R\left(e_{1}, e_{5}\right) e_{1}=e_{5}, R\left(e_{1}, e_{5}\right) e_{5}=-e_{1}, \\
& R\left(e_{2}, e_{3}\right) e_{2}=e_{3}, R\left(e_{2}, e_{3}\right) e_{3}=-e_{2}, R\left(e_{2}, e_{4}\right) e_{2}=e_{4}, R\left(e_{2}, e_{4}\right) e_{4}=-e_{2}, \\
& R\left(e_{2}, e_{5}\right) e_{2}=e_{5}, R\left(e_{2}, e_{5}\right) e_{5}=-e_{2}, R\left(e_{3}, e_{4}\right) e_{3}=e_{4}, R\left(e_{3}, e_{4}\right) e_{4}=-e_{3}, \\
& R\left(e_{3}, e_{5}\right) e_{3}=e_{5}, R\left(e_{3}, e_{5}\right) e_{5}=-e_{3}, R\left(e_{4}, e_{5}\right) e_{4}=e_{5}, R\left(e_{4}, e_{5}\right) e_{5}=-e_{4} .
\end{aligned}
$$

and

$$
\begin{aligned}
& \bar{R}\left(e_{1}, e_{2}\right) e_{1}=2 e_{2}, \bar{R}\left(e_{1}, e_{2}\right) e_{2}=-2 e_{1}, \bar{R}\left(e_{1}, e_{3}\right) e_{1}=2 e_{3}, \bar{R}\left(e_{1}, e_{3}\right) e_{3}=-2 e_{1}, \\
& \bar{R}\left(e_{1}, e_{4}\right) e_{1}=2 e_{4}, \bar{R}\left(e_{1}, e_{4}\right) e_{4}=-2 e_{1}, \bar{R}\left(e_{1}, e_{5}\right) e_{1}=2 e_{5}, \bar{R}\left(e_{1}, e_{5}\right) e_{5}=-2 e_{1}, \\
& \bar{R}\left(e_{2}, e_{3}\right) e_{2}=2 e_{3}, \bar{R}\left(e_{2}, e_{3}\right) e_{3}=-2 e_{2}, \bar{R}\left(e_{2}, e_{4}\right) e_{2}=2 e_{4}, \bar{R}\left(e_{2}, e_{4}\right) e_{4}=-2 e_{2}, \\
& \bar{R}\left(e_{2}, e_{5}\right) e_{2}=2 e_{5}, \bar{R}\left(e_{2}, e_{5}\right) e_{5}=-2 e_{2}, \bar{R}\left(e_{3}, e_{4}\right) e_{3}=2 e_{4}, \bar{R}\left(e_{3}, e_{4}\right) e_{4}=-2 e_{3}, \\
& \bar{R}\left(e_{3}, e_{5}\right) e_{3}=2 e_{5}, \bar{R}\left(e_{3}, e_{5}\right) e_{5}=-2 e_{3}, \bar{R}\left(e_{4}, e_{5}\right) e_{4}=2 e_{5}, \bar{R}\left(e_{4}, e_{5}\right) e_{5}=-2 e_{4} .
\end{aligned}
$$

With the help of the above curvature tensors with respect to the quarter-symmetric metric connection we find the Ricci tensors [12] as follows:

$$
\bar{S}\left(e_{1}, e_{1}\right)=\bar{S}\left(e_{2}, e_{2}\right)=\bar{S}\left(e_{3}, e_{3}\right)=\bar{S}\left(e_{4}, e_{4}\right)=\bar{S}\left(e_{5}, e_{5}\right)=-8 .
$$

Also it follows that the scalar curvature tensor [12] with respect to the quartersymmetric metric connection is $\bar{r}=-40$.

Let $X, Y, Z$ and $U$ be any four vector fields given by

$$
\begin{gathered}
X=a_{1} e_{1}+a_{2} e_{2}+a_{3} e_{3}+a_{4} e_{4}+a_{5} e_{5}, \quad Y=b_{1} e_{1}+b_{2} e_{2}+b_{3} e_{3}+b_{4} e_{4}+b_{5} e_{5} \\
Z=c_{1} e_{1}+c_{2} e_{2}+c_{3} e_{3}+c_{4} e_{4}+c_{5} e_{5} \quad W=d_{1} e_{1}+d_{2} e_{2}+d_{3} e_{3}+d_{4} e_{4}+d_{5} e_{5},
\end{gathered}
$$

where $a_{i}, b_{i}, c_{i}, d_{i}$, for all $i=1,2,3,4,5$ are all non-zero real numbers.

Using the above curvature tensors and the scalar curvature tensors of the quartersymmetric metric connection, we obtain

$$
\begin{aligned}
\overline{\mathbb{W}}(X, Y) \xi= & {\left[\left(2 e_{2}-2 e_{2}\right)\left(a_{1} b_{2}\right)+\left(2 e_{5}-2 e_{5}\right)\left(a_{1} b_{5}\right)\right.} \\
& \left.+\left(2 e_{4}-2 e_{4}\right)\left(a_{1} b_{4}\right)+\left(2 e_{3}-2 e_{3}\right)\left(a_{1} b_{3}\right)\right]=0,
\end{aligned}
$$


which verifies the result of Section 3 .

Now we see that the $\phi$-concircularly flat with respect to the quarter-symmetric metric connections from the above relations as follow:

$$
\begin{aligned}
\overline{\mathbb{W}}(\phi X, \phi Y, \phi Z, \phi U)= & 2 a_{2} b_{3}\left(c_{2} d_{3}-c_{3} d_{2}\right)+2 a_{2} b_{5}\left(c_{2} d_{5}-c_{5} d_{2}\right)+2 a_{3} b_{4}\left(c_{3} d_{4}-c_{4} d_{3}\right) \\
& +2 a_{4} b_{5}\left(c_{4} d_{5}-c_{5} d_{4}\right)+2 a_{2} b_{4}\left(c_{2} d_{4}-c_{4} d_{2}\right) \\
& +2 a_{3} b_{5}\left(c_{3} d_{5}-c_{5} d_{3}\right)=0 .
\end{aligned}
$$

Hence P-Sasakian manifolds will be $\phi$-concircularly flat with respect to the quartersymmetric metric connections if $\frac{c_{2}}{d_{2}}=\frac{c_{3}}{d_{3}}=\frac{c_{4}}{d_{4}}=\frac{c_{5}}{d_{5}}$.

The above arguments tell us that the 5-dimensional P-Sasakian manifolds with respect to the quarter-symmetric metric connections under consideration agrees with the Section 4.

Acknowledgements. The author is thankful to the referee for his/her valuable suggestions for the improvement of the paper.

\section{REFERENCES}

[1] T. Adati and K. Matsumoto, On conformally recurrent and conformally symmetric P-Sasakian manifolds, Turkish J. Math. 13 (1977), 25-32.

[2] A. Barman, Semi-symmetric non-metric connection in a P-Sasakian manifold, Novi Sad J. Math. 43 (2013), 117-124.

[3] A. Barman, On Para-Sasakian manifolds admitting semi-symmetric metric connection, Publ. Inst. Math. (Beogard) (N.S.) 95 (2014), 239-247.

[4] A. Barman and G. Ghosh, Concircular curvature tensor of a semi-symmetric non-metric connection on P-Sasakian manifolds, An. Univ. Vest. Timis. Ser. Mat.-Inform. LIV (2016), 47-58.

[5] D. E. Blair, Inversion theory and conformal mapping, Stud. Math. Libr. 9, Amer. Math. Soc. (2000).

[6] U. C. De and G. Pathak, On P-Sasakian manifolds satisfying certain conditions, J. Indian Math. Soc. (N.S.) 16 (1994), 72-77.

[7] U. C. De, C. Özgür, K. Arslan, C. Murathan and A. Yildiz, On a type of P-Sasakian manifolds, Math. Balkanica (N.S.) 22 (2008), 25-36.

[8] S. Desmukh and S. Ahmed, Para-Sasakian manifolds isometrically immersed in spaces of constant curvature, Kyungpook Math. J. 20 (1980), 112-121.

[9] A. Friedmann and J. A. Schouten, Über die Geometric der halbsymmetrischen Übertragung, Math. Z. 21 (1924), 211-223.

[10] S. Golab, On semi-symmetric and quarter-symmetric liner connections, Tensor (N.S.) 29 (1975), 249-254.

[11] W. Kuhnel, Conformal Transformations between Einstein Spaces, Bonn, 1985/1986, 105-146, Aspects Math. E12, Vieweg, Braunschweig, 1988.

[12] K. Mandal and U. C. De, Quarter-symmetric metric connection in a P-Sasakian manifold, An. Univ. Vest. Timis. Ser. Mat.-Inform. LIII (2015), 137-150.

[13] K. Matsumoto, S. Ianus and I. Mihai, On a P-Sasakian manifolds which admit certain tensor fields, Publ. Math. Debrecen 33 (1986), 61-65.

[14] I. Sato, On a structure similar to the almost contact structure, Tensor (N.S.) 30 (1976), 219-224.

[15] K. Yano, Concircular Geometry I. Concircular Transformations, Proc. Imp. Acad. Tokyo 16 (1940), 195-200. 
[16] K. Yano and S. Bochner, Curvature and Betti numbers, Ann. of Math. Stud. 32 (1953).

[17] A. Yildiz, M. Turan and B. E. Acet, On Para-Sasakian manifolds, Dumlupinar Üniversitesi 24 (2011), 27-34.

Department of Mathematics

Ramthakur College

P.O.-Arundhuti Nagar-799003, Dist.-West Tripura, Tripura, India

Email address: ajitbarmanaw@yahoo.in 